\title{
Streamlining the Generation of an Osteogenic Graft by 3D Culture of Unprocessed Bone Marrow on Ceramic Scaffolds
}

\author{
Anindita Chatterjea ${ }^{* 1}$, Auke J.S. Renard ${ }^{2}$, Christel Jolink ${ }^{3}$, Clemens A. van Blitterswijk ${ }^{1}$, \\ Jan de Boer ${ }^{1}$ \\ ${ }^{1}$ University of Twente, MIRA Institute for Biomedical Technology and Technical \\ Medicine, Department of Tissue Regeneration, Enschede, The Netherlands; ${ }^{2}$ Department \\ of Orthopaedic Surgery, Medisch Spectrum Twente Hospital, Enschede, The \\ Netherlands; ${ }^{3}$ Laboratorium Pathologie Oost Nederland, Enschede, The Netherlands
}

\begin{abstract}
Mesenchymal stromal cells are present in very low numbers in the bone marrow, necessitating their selective expansion on tissue culture plastic prior to their use in tissue engineering applications. MSC expansion is laborious, time consuming, unphysiological and not economical, thus calling for automated bioreactor-based strategies. We and others have shown that osteogenic grafts can be cultured in bioreactors by seeding either 2D - expanded cells or by direct seeding of the mononuclear fraction of bone marrow. To further streamline this protocol, we assessed in this manuscript the possibility to seed the cells onto porous calcium phosphate ceramics directly from unprocessed bone marrow. Using predetermined volumes of bone marrow from multiple human donors with different nucleated cell counts, we were able to grow a confluent cell sheath on the
\end{abstract}


scaffold surface in 3 weeks. Cells of both stromal, endothelial and haematopoietic origin were detected, in contrast to grafts grown from 2D expanded cells where only stromal cells could be seen. Upon implantation in nude mice, similar quantities of bone tissue were generated as compared to that obtained by using the conventional number of culture expanded cells from the same donor. We conclude that human osteogenic grafts can be efficiently prepared by direct seeding of cells from unprocessed bone marrow.

Key words: Mesenchymal Stromal Cells, Bone tissue engineering, Osteogenic grafts, Unprocessed bone marrow, 3D culture, Translational research

\section{Introduction}

Adult mesenchymal stem or stromal cells (MSCs) obtained from bone marrow have great potential in tissue engineering applications as they can be easily isolated and differentiated into osteogenic, chondrogenic and adipogenic lineages. [1] [2] [3]. One of the many tissue engineering applications is the field of bone defect reconstruction. For this application, MSCs are differentiated into osteoblasts in vitro as evidenced by the deposition of a mineralized matrix and expression of osteogenic markers such as alkaline phosphatase (ALP). They are then implanted in vivo to bridge bone defects [4] [5] [6] [7]. However, MSCs represent a rare population of cells with a reported incidence of 0.01 to $0.001 \%$ within the bone marrow [8] [1] and large numbers are required to heal bone defects. For instance, spinal fusion surgery requires a minimum of $4 \mathrm{cc}$ of graft material when performed using the anterior interbody fusion method [9]. In our lab, we typically use porous calcium phosphate ceramics, $2-3 \mathrm{~mm}$ in size with 200,000 cells per 
particle to generate an osteogenic graft. To generate $4 \mathrm{cc}$ of graft material, at least 36 million cells would be required. The need for such large cell numbers coupled with the low frequency of MSCs in bone marrow, necessitates their expansion prior to their use in bone tissue engineering and also other applications. Isolation and expansion of MSCs relies on their ability to adhere to plastic [10]. When bone marrow is plated onto tissue culture plastic, a population of cells is obtained which is referred to as MSCs, based on their multipotency and CD expression profile. MSCs are then further expanded to obtain a sufficient number of cells to load on scaffolds for tissue engineering applications [3] [6] [11].

However, it is well recognized that culturing cells in a monolayer on plastic, bereft of the company of the heterogeneous cell populations normally present within the bone marrow, is not physiological. The monolayer culture does not provide an ideal milieu for cell-cell and cell-extracellular matrix interactions, thereby limiting the mechanical and biochemical cues required for the optimal functioning of the cells [12] [13] [14].

The number of clonogenic MSCs present in bone marrow varies greatly from donor to donor. For instance, we tested 27 donors with varying nucleated counts. On plating the aspirates on tissue culture plastic at a density of 500,000 mononuclear cells $/ \mathrm{cm}^{2}$ and trypsinizing them at $80 \%$ confluency, we obtained on average, 4 million MSCs (data not shown). As previously mentioned, 9 million cells are required for obtaining 1cc of graft material and for clinical application at least 30-40 million cells are required. Thus, in the conventional method of tissue engineering using tissue culture plastic, multiple cycles of 
medium change, cell trypsinizing, counting and replating are required to obtain a sufficient number of cells. Semi-automation of the process to engineer bone grafts using bioreactor technology is under investigation [14-22]. Here, MSCs are isolated from the bone marrow and expanded in $2 \mathrm{D}$ to obtain the required numbers. Next, seeding and proliferation on scaffolds are performed within the bioreactor. Bioreactors provide a closed standardized culture system which requires minimal operator handling and good physicochemical environmental control, which is crucial for cell survival and proliferation. However, the $2 \mathrm{D}$ expansion phase still presents a barrier to complete automation. Based on these considerations, researchers have tried to culture bone marrow- derived MSCs directly on 3D scaffolds, bypassing the need for the 2D expansion phase [23] [24] [25] [26]. The nucleated cells within the bone marrow were separated using a density gradient separation method. Then, mononuclear cells were seeded in the bioreactor on scaffolds to grow the cell ceramic constructs. These studies demonstrated that osteoinductive grafts could be generated within an exclusive 3D system with results comparable to the conventional 2D method of generating grafts [23] [24]. This possibility of expanding MSCs within 3D scaffolds opens new frontiers in the streamlining of the process for therapeutic use.

Whereas the mononuclear cell count is typically used to express the cellularity of a bone marrow aspirate, it does not accurately represent the number of colony forming units (CFU-Fs) contained within the particular marrow [27]. The best predictor of the osteogenic capacity of cell-laden scaffold constructs is the estimation of the final number of clonogenic MSCs implanted [23]. Cell surface markers such as STRO-1 and nerve 
growth factor (NGF) receptor may be used to prospectively isolate clonogenic MSCs from the crude marrow $[28,29]$. However, for cell isolation the marrow is subjected to cell separation strategies, which is again labour intensive. Based on this, we decided on another approach to standardize and streamline the generation of an osteogenic graft.

The aim of the work described in this manuscript is to further streamline the generation of osteogenic grafts by using volume of bone marrow rather than the concentration of nucleated cells in the bone marrow as a guiding parameter. This facilitates the clinical translation without affecting the bone forming potential of the engineered constructs. We demonstrate that within the same total culture time frame, a defined volume of fresh unprocessed bone marrow seeded directly on scaffolds in a static set up, could reproducibly produce grafts with similar osteogenic potential as those obtained by seeding and culturing 2D expanded cells. 


\section{Materials and methods}

\subsection{Bone marrow aspirates}

Bone marrow aspirates (10-25 $\mathrm{ml}$ total volume) were obtained from 4 healthy donors during hip replacement surgery (donor information in Table 1) with written informed consent. Part of the bone marrow was used to isolate and proliferate hMSCs in $2 \mathrm{D}$ on tissue culture plastic [30] while the rest of the bone marrow was directly seeded onto the scaffolds.

\section{2. $2 D$ isolation and expansion of cells}

hMSCs were isolated and proliferated from the start of the culture period in hMSC osteogenic medium comprising ( $\alpha$-Modified Eagles Medium, Gibco) supplemented with $10 \%$ foetal bovine serum (FBS, Lonza), $0.2 \mathrm{mM}$ ascorbic acid (Sigma), $2 \mathrm{mM} \mathrm{L-}$ glutamine (Gibco), 100 units/ml Penicillin (Gibco), $100 \mu \mathrm{g} / \mathrm{ml}$ Streptomycin (Gibco) and $10^{-8} \mathrm{M}$ dexamethasone (Sigma) to commit the cells towards the osteogenic lineage [31]. To isolate the hMSCs on tissue culture plastic, aspirates were resuspended using a 20 gauge needle, plated on tissue culture flasks at a density of 500,000 cells per square centimetre and cultured in hMSC osteogenic medium. Cells were grown at $37^{\circ} \mathrm{C}$ in a humid atmosphere with $5 \% \mathrm{CO}_{2}$. Medium was changed after 5 days at which moment all the non-attached cells were removed. Thereafter, medium was refreshed twice a week for a total period of 2 weeks. The cells were then trypsinised, counted and seeded at a density of 200,000 cells/porous biphasic calcium phosphate scaffolds (kindly provided by Dr. Huipin Yuan, University of Twente, The Netherlands) produced according to the $\mathrm{H}_{2} \mathrm{O}_{2}$ 
method including naphthalene as described previously [32]. The material was sintered at $1300{ }^{\circ} \mathrm{C}$. The average size of the granules was $2-3 \mathrm{~mm}$ with the specific surface area being $0.2 \mathrm{~m}^{2} / \mathrm{g}$. The composition of the particles is 20TCP/80HA. The microporosity (volume percentage of micropores smaller than $10 \mu \mathrm{m}$ within the ceramic) is $8.7 \%$ while the calcium release is $4.2 \pm 0.4 \mathrm{ppm}$.

\subsection{Direct seeding of bone marrow on scaffolds}

For direct seeding, $200 \mu \mathrm{l}$ of unprocessed bone marrow was gently dispersed over the surface of 3, 2-3 mm (BCP) scaffolds. After 4 hours, $2 \mathrm{ml}$ of osteogenic medium was slowly added to each set of 3 scaffolds. Medium was changed after 5 days, and the cells were cultured for 3 weeks on the BCP scaffolds with regular medium change. As a control, expanded cells from the same donor were trypsinised after a 2 week culture period on tissue culture plastic. $200 \mu \mathrm{l}$ aliquots were then made such that each aliquot contained 600,000 cells. The cells were then dispersed slowly over the surface of the 3 scaffolds. After 4 hours, osteogenic medium was slowly added to the scaffolds. The expanded cells were cultured on these scaffolds for a period of 1 week with one medium change after 3 days.

\subsection{Cell proliferation, distribution, viability and cell morphology on scaffolds}

Cell numbers on the scaffolds were qualitatively assessed at the end of week 1,2 and 3 of the culture period by methylene blue (MB) staining. Cells were fixed with $1.5 \%$ glutaraldehyde in $0.14 \mathrm{M}$ cacodylic buffer, $\mathrm{pH}$ 7.3. After fixation, $1 \% \mathrm{MB}$ solution (Sigma) was added and incubated for 60 seconds. The scaffolds were washed twice with 
PBS in order to remove non-bound MB. Attached cells were visualized using light microscopy. A quantitative assessment of the number of cells on scaffolds was obtained by determining the DNA content on the scaffolds from both conditions using the Cyquant cell proliferation assay kit (Molecular Probes, Invitrogen detection techniques) according to the manufacturer's instructions. For cell viability 3-(4, 5-dimethylthiazol-2-yl)-2, 5diphenyltetrazolium bromide (MTT) (Sigma) staining was used. A solution of 1\% MTT was applied to the scaffolds containing cells. After $4 \mathrm{~h}$ of incubation, the MTT solution was removed by flushing the scaffolds with PBS. Scaffolds and cells were visualized using light microscopy. The morphology of the cells was studied using scanning electron microscopy (SEM). Scaffolds seeded directly with bone marrow and with 2D expanded cells were fixed in $4 \%$ paraformaldehyde, dehydrated with a series of graded ethanol washes and critical point dried with liquid $\mathrm{CO}_{2}$. Fixed scaffolds were sputtered with gold and examined.

\subsection{Cell Characterization}

The cells were characterized by immunostaining with antibodies against CD105 (Endoglin; Dako), CD31 (PECAM-1; Dako) and CD45 (leukocyte common antigen; Dako). The scaffolds were fixed in $10 \%$ formalin, embedded in agarose, decalcified using $2 \%$ formic acid for 24 hours and then embedded in paraffin. The paraffin samples were sectioned at $5 \mu \mathrm{m}$, mounted to the slide, deparaffinised and rehydrated. For staining with $\mathrm{CD} 31$, antigen retrieval was performed by boiling the slides in sodium citrate buffer $\mathrm{pH}$ 6.0 for 20 minutes. No antigen retrieval was required for CD45 whereas pre-treatment of the sections with proteolytic enzymes was performed prior to staining with CD105. The 
sections were blocked using 1\% BSA (Sigma) in PBS. Primary antibody incubation was performed for 1 hour in humidified chambers using 1:10 dilution of either monoclonal mouse anti-human CD105, CD31 or CD45. Next, the endogenous peroxidise activity was blocked and the slides were incubated with goat anti mouse HRP conjugated secondary $\mathrm{AB}$ (Immunologic). The cells were counterstained with haematoxylin and eosin to stain the cytoplasm and nuclei of cells. The slides were visualised with a light microscope (Leica). Immunostaining was performed on a total of 54 sections obtained from scaffolds seeded directly and with 2D expanded cells from donor 1,2 and 3. Thus, there were 3 sections per antibody per condition for each of the 3 donors.

\subsection{In Vivo studies}

To compare the amount of bone formed by either direct seeding of bone marrow or seeding of 2D - expanded bone marrow cells, in vivo studies were designed in ectopic locations in immune-deficient mice, a model widely used for assessing bone forming capacity of hMSCs [7, 33-36]. The total culture time for the cells in both the direct seeding and the 2D expanded seeding was kept at 3 weeks. In the former, the cells were on the scaffolds from the start while in the latter they were first cultured for 2 weeks on plastic and then transferred to the scaffolds. Prior to in vivo implantation, some scaffolds were used to stain with $\mathrm{MB}$ to confirm the presence of cells on the surface of the scaffolds. Six immune-deficient male mice (Hsd-cpb:NMRI-nu, Harlan) were used for the first donor and 6 immune-deficient male mice (Crl:NMRI-Foxn1-nu-,Charles river) were used for each of the last three donors. The mice were anesthetized by inhalation of isoflurane and oxygen. Two subcutaneous pockets were made and each pocket was 
implanted with 3 scaffolds, of each condition. The incisions were closed using a vicryl 50 suture. After 6 weeks the mice were sacrificed using $\mathrm{CO}_{2}$ and samples were explanted. The experiments were approved by the local animal experimental committee.

\subsection{Bone Histomorphometry}

The explanted samples were fixed in $4 \%$ paraformaldehyde and embedded in methacrylate (L.T.I, Bilthoven, The Netherlands) for sectioning. Approximately $300 \mu \mathrm{m}$ thick, undecalcified sections were processed on a histological diamond saw (Leica saw microtome cutting system). At least 6 sections were made from each sample and the sections were stained with basic fuchsin and MB to visualize new bone formation. The newly formed mineralized bone stains red with basic fuchsin while all other cellular tissues stain light blue with $\mathrm{MB}$, and the ceramic material remains black and unstained by both the dyes. Histological sections were qualitatively analysed using light microscopy (Leica), and each histological section was scored either positive or negative for bone formation. Quantitative histomorphometry was performed as described previously [7]. Briefly, sections were scanned using Minolta Dimage Scan and high-resolution digital photographs (300 dpi) were made from three randomly selected sections from each tissue-engineered graft. For histomorphometrical analysis, bone and material were pseudo-coloured green and red, respectively, using Photoshop CS2 (Adobe Systems). Image analysis was performed using a PC-based system with KS400 software (version 3, Zeiss). A custom-made programme was used to measure bone/ceramic surface ratios. 


\section{Results}

We were interested in assessing the feasibility of using unprocessed bone marrow for direct seeding on scaffolds for bone tissue engineering applications. To do so, we performed studies to determine the volume of unprocessed fresh bone marrow, which when cultured on scaffolds for 3 weeks, reliably and reproducibly generated tissue engineered constructs with cell numbers and in vivo bone forming potential similar to that obtained by seeding $2 \mathrm{D}$ expanded cells. For all the studies performed, our aim was to have a scaffold covered with a confluent layer of cells.

\section{1. hMSC expansion on 3D scaffolds}

We first aimed at determining the possibility of growing hMSCs directly on scaffolds using unprocessed fresh bone marrow. To do this, we dispersed $1 \mathrm{ml}$ of fresh, unmanipulated bone marrow directly onto 3 BCP scaffolds. After a week, we examined the presence of cells using MB and tested their viability using a MTT assay. Already after one week of culture, we observed foci of cells growing on the ceramics (fig 1A) and after three weeks of culture, the surface of the scaffold was completely covered by viable cells (Fig 1C, Fig 1D) similar to the condition where expanded cells were allowed to grow on the scaffolds for one week (Fig 1E, F). Our results show that it is possible to grow cells directly on scaffolds using fresh unmanipulated bone marrow. We then tried to determine the amount of bone marrow required to yield a cell sheath on the scaffolds after 3 weeks in culture. We chose a 3 week period because that is the time required to produce an osteogenic graft with 2 weeks of culture on plastic and one week on the scaffolds. We conducted studies with hMSCs derived from 8 different donors and the volumes of bone 
marrow used for seeding ranged from $50 \mu \mathrm{l}$ to $1 \mathrm{ml}$ with varying concentrations of nucleated cell counts (see table 2). Different volumes of bone marrow from different donors are dispersed over the scaffolds and after an initial 4hours incubation period the osteogenic medium was added. The scaffolds were left in culture for a period of 3 weeks. Cells were stained at weekly intervals (Fig 2). An average of $200 \mu \mathrm{l}$ of bone marrow per 3 scaffolds produced a confluent cell sheath. Lower volumes did not form a cell sheath in 3 weeks while using higher volumes either did not provide significant benefits or led to detachment of the cell sheath from the scaffold surface (Fig 2)

Based on these results, we used $200 \mu 1$ of unprocessed bone marrow per 3 scaffolds with a 3 week culture period for all our experiments. To compare the direct seeding method with the conventional approach using 2D expanded cells, 600,000 2D expanded cells were seeded per 3 scaffolds. In our experience, this number results in a confluent layer of cells on the scaffolds after 1 week $[11,37]$.

\subsection{Cell quantification and viability}

We quantified the number of cells present on the scaffolds seeded directly with bone marrow to those seeded with 2D expanded cells using the Cyquant assay. In two of the three donors tested, the number of cells was similar on both scaffolds (Fig 3). In only one of the donors, two times more cells were found on the scaffolds seeded with $2 \mathrm{D}$ expanded cells as compared to those seeded directly with bone marrow. In all the 4 donors, the viability of the cells present on the scaffolds was tested using the MTT assay. 
This test confirmed that most of the cells in all 4 donors and in both conditions were metabolically active at the time of in vivo implantation (Fig 1D, 1F).

\subsection{Cell characterization}

Scanning electron microscopy of the ceramics at the end of the 3 week culture period demonstrated that all cells on the scaffolds seeded with $2 \mathrm{D}$ expanded cells had a fibroblastic morphology (Fig 4B). In contrast, on the scaffolds directly seeded with bone marrow, we noted the presence of spheroidal cells interspersed by predominantly fibroblastic cells (data not shown), suggesting that during direct seeding of bone marrow, populations of cells other than MSCs grew on the scaffold. To identify the population of cells on the ceramics from both groups, we performed immunostaining using antibodies against cells of mesenchymal (CD105), endothelial (CD31) and hematopoietic (CD45) lineage. For immunostaining, 3 scaffolds were included in the direct seeded and 2D expanded groups each and sections were obtained from each scaffold. 3 sections from every scaffold were stained for each of the 3 antibodies, i.e. CD31, CD45 and CD105. In the sections from scaffolds seeded with 2D expanded cells, we observed no positive staining for CD31 or CD45 (Fig 4c). When stained with the CD 105 antibody, we observed a sheath of positively stained cells, indicating that all the cells on the scaffold were of mesenchymal origin (Fig 4d). In contrast, on scaffolds seeded directly with bone marrow we observed on average, 2-3 clusters of 3-4 cells positive for CD45 in each of the scaffold sections stained for CD45 (Fig 4a). CD31 positive cells, however, were not observed in all sections. In the sections stained for CD31, 0-2 CD31 positive cells were observed per section (Fig 4b). However, the majority of the cells on the direct seeded 
scaffolds stained positive for CD105. In conclusion, as compared to scaffolds seeded with 2D expanded cells, directly seeded scaffolds have a more heterogeneous cell population. However, in spite of the heterogeneity, the majority of the cells on the directly seeded scaffolds are MSCs, confirming our hypothesis that despite the very low numbers of MSCs in unprocessed bone marrow, this population of cells directly adheres and expands on the scaffold surface

\subsection{In Vivo Bone Formation}

To evaluate the bone forming capacity of the constructs, we implanted them for a period of 6 weeks in subcutaneous pockets in immune-deficient mice. Upon explantation, bone formation was analysed histomorphometrically. In the explanted samples from all 4 donors, except in those obtained from direct seeding of bone marrow from donor 1 , histological examination revealed the presence of bone tissue, in which we observed osteocytes embedded in a mineralized extracellular matrix (Fig 5A). As seen before, the amount of bone obtained differs between different donors [11]. In donor 2, the bone/ceramic surface ratio increased from $0.14 \%$ in the scaffolds seeded with $2 \mathrm{D}$ expanded cells to $0.6 \%$ in those seeded directly with bone marrow and in donor 3 from $0.20 \%$ in scaffolds seeded with $2 \mathrm{D}$ expanded cells to $0.37 \%$ in those seeded directly with bone marrow (Fig 5B). However, the results were not statistically significant when compared with Student's paired t test $(\mathrm{p}>0.05 \%)$. Donor 4 performed the best in terms of bone formation. In this donor, the bone/ceramic surface ratio significantly increased from $2.6 \%$ in the scaffolds seeded with $2 \mathrm{D}$ expanded cells to $7.7 \%$ in the scaffolds directly seeded with bone marrow (Fig 5B). This donor showed a significant benefit from the 
direct seeding approach ( $\mathrm{p}<0.05 \%$ using Student's paired t test). When data from all the 4 donors was combined, the average percentage bone/ceramic surface ratio increased from 0.67 to 2.18 . Although not statistically significant, the direct seeding method shows a trend toward increased bone formation in vivo compared to the 2D method. For practical purposes, the results indicate that the direct seeding method using unprocessed bone marrow is at least as efficient in generating bone tissue upon implantation. 


\section{Discussion}

In the present study, we provide proof of principle on using pre-determined volumes of unprocessed bone marrow to generate grafts which have similar osteo-inductive potential as those produced within the same culture period, by seeding of MSCs derived from the same donor using the labour intensive 2D expansion.

In 1987, Friedenstein et al. demonstrated the osteogenic potential of bone marrow derived MSCs, which led to the idea that hMSCs can be used to regenerate bone defects. In spite of all the interest this idea generated, a quarter of a century later, hMSCs are still not available to the surgeons as a routine off the shelf treatment option [38]. One of the plausible reasons is that human bone marrow derived MSCs have very high donor to donor variability which cannot be predicted a priori. This translated into problems obtaining reproducible amounts of bone using MSCs, both in ectopic and orthotopic locations, thus limiting their use in clinical trials. The other limiting factor is the lack of standardized methods to generate grafts which, in addition to being cost efficient, are user friendly and do not require laboratory trained manpower and expensive equipment within the hospital set up. In this study we tried to address the second limiting factor. Proof of principle was found previously, when nucleated cell from minimally processed marrow was seeded onto scaffolds within a closed bioreactor with ectopic bone formation in vivo $[23,24]$. Our approach of seeding scaffolds directly with unprocessed pre-determined volumes of bone marrow simplifies the process of generating osteogenic grafts even further. A similar approach of directly seeding bone marrow on scaffolds was also used 
by other researchers in the past to generate bone in a critical size defect with limited success [39] [40]. One of the main differences in their strategy as compared to ours was that in all these studies the scaffolds seeded with bone marrow were implanted within an hour (referred to as per-operative cell seeding). We have already demonstrated the poor performance of per-operative seeding even with 2D expanded cells [40]. Evidently expansion of MSCs prior to implantation is necessary. In this manuscript, MSC expansion occurred on scaffolds in a static environment in well plates whereas bioreactors were used by previous researchers to culture the scaffolds in a dynamic environment. Although commercially viable, automated systems are still not available for routine hospital use [41], the direct seeding approach can be adapted for use within a bioreactor set up.

Previously researchers used nucleated cell counts as a guide to assess the population of CFU-Fs in the bone marrow. In our in vivo study, instead of using nucleated cell counts, an average volume of bone marrow was used in all the four donors as a practical readout. Although the nucleated counts were obtained, they were not taken into consideration when seeding the scaffold. In retrospect, we observe that donor 1 which had the lowest nucleated cell count (Table 1) performed worst in the in vivo setting and donor 4 with the highest nucleated cell count performed the best. In contrast, although donor 3 had a much higher nucleated cell count than donor 2, still directly seeded scaffolds in donor 2 gave more bone than donor 3. In general there was no definite correlation between the amount of bone formed and the initial number of nucleated cells in the bone marrow. This is in agreement with literature, which suggests that nucleated cell counts do not indicate CFU- 
Fs present in a particular amount of bone marrow [42]. One may argue that a predetermined amount of bone marrow is also not a fool proof method of ensuring a cell scaffold construct with osteogenic potential thereby warranting additional quality control criteria in future studies. Markers such as STRO-1 and NGF currently available may prospectively determine the number of CFU-Fs in the bone marrow. Nevertheless, in our study using predetermined volumes of bone marrow as in the 8 donors with varying nucleated cell counts that we studied, we were able to obtain a complete coverage of the scaffolds in 3 weeks using $200 \mu 1$ of bone marrow per 3 scaffolds, in all the donors.

It can be argued that using the $2 \mathrm{D}$ expansion method for greater than 2 weeks as used in this study, a larger number of cells could be obtained and this in turn could support generation of larger osteoinductive constructs. However it has been reported in literature that 2D expanded bone marrow derived hMSCs have a much lower differentiation capacity as compared to the MSCs found in fresh bone marrow. Repeated passaging of cells can eventually lead to their senescence [43-45]. Moreover, $20-40 \mathrm{ml}$ of bone marrow can be obtained safely from patients [30]. This can generate as much as 8 to 13 cc of graft material which would be sufficient for most routine clinical applications. Further, here we chose a period of 3 weeks as the culture period as we wanted to compare our findings with scaffolds seeded with 2D expanded cells. However, depending on the application, the culture period and the amount of bone marrow used per scaffold can be modified. 
Based on the results of this study and using this approach of direct seeding, we are now testing a prototype of a compact, closed, sterile system which is pre-packed with scaffolds and which could be used in the clinics without the need for trained personnel or special sterile work areas. This device in combination with the direct seeding approach can thus be utilised by a surgeon directly within the surgical theatre and then left for the culture duration in an incubator routinely present in most hospitals.

In conclusion, the direct seeding approach offers potential use in clinical situations. However, the osteogenicity of the grafts either using the conventional approach or the approach proposed in this work is not comparable as yet to the autologous bone grafts or that observed with MSCs derived from rat or goat. Further studies using supplementation of the medium with osteogenic factors should be considered to help address this issue. 


\section{References}

1. Pittenger, M.F., et al., Multilineage potential of adult human mesenchymal stem cells. Science, 1999. 284(5411): p. 143-7.

2. Bianco, P., et al., Bone marrow stromal stem cells: nature, biology, and potential applications. Stem Cells, 2001. 19(3): p. 180-92.

3. Friedenstein, A.J., R.K. Chailakhjan, and K.S. Lalykina, The development of fibroblast colonies in monolayer cultures of guinea-pig bone marrow and spleen cells. Cell Tissue Kinet, 1970. 3(4): p. 393-403.

4. Meijer, G.J., et al., Cell based bone tissue engineering in jaw defects. Biomaterials, 2008. 29(21): p. 3053-61.

5. Viateau, V., et al., Long-bone critical-size defects treated with tissue-engineered grafts: a study on sheep. J Orthop Res, 2007. 25(6): p. 741-9.

6. Haynesworth, S.E., et al., Characterization of cells with osteogenic potential from human marrow. Bone, 1992. 13(1): p. 81-8.

7. Siddappa, R., et al., cAMP/PKA pathway activation in human mesenchymal stem cells in vitro results in robust bone formation in vivo. Proc Natl Acad Sci U S A, 2008. 105(20): p. 7281-6.

8. Friedenstein, A.J., R.K. Chailakhyan, and U.V. Gerasimov, Bone marrow osteogenic stem cells: in vitro cultivation and transplantation in diffusion chambers. Cell Tissue Kinet, 1987. 20(3): p. 263-72.

9. Kruyt, M.C., et al., Bone tissue engineering and spinal fusion: the potential of hybrid constructs by combining osteoprogenitor cells and scaffolds. Biomaterials, 2004. 25(9): p. 1463-73.

10. Dominici, M., et al., Minimal criteria for defining multipotent mesenchymal stromal cells. The International Society for Cellular Therapy position statement. Cytotherapy, 2006. 8(4): p. 315-7.

11. Siddappa, R., et al., Donor variation and loss of multipotency during in vitro expansion of human mesenchymal stem cells for bone tissue engineering. J Orthop Res, 2007. 25(8): p. 1029-41.

12. Abbott, A., Cell culture: biology's new dimension. Nature, 2003. 424(6951): p. 870-2.

13. Lund, A.W., et al., The natural and engineered 3D microenvironment as a regulatory cue during stem cell fate determination. Tissue Eng Part B Rev, 2009. 15(3): p. 371-80.

14. Timmins, N.E., et al., Three-dimensional cell culture and tissue engineering in a T-CUP (tissue culture under perfusion). Tissue Eng, 2007. 13(8): p. 2021-8.

15. Janssen, F.W., et al., Human tissue-engineered bone produced in clinically relevant amounts using a semi-automated perfusion bioreactor system: a preliminary study. J Tissue Eng Regen Med, 2010. 4(1): p. 12-24.

16. Janssen, F.W., et al., A perfusion bioreactor system capable of producing clinically relevant volumes of tissue-engineered bone: in vivo bone formation showing proof of concept. Biomaterials, 2006. 27(3): p. 315-23.

17. Wendt, D., et al., Oscillating perfusion of cell suspensions through threedimensional scaffolds enhances cell seeding efficiency and uniformity. Biotechnol Bioeng, 2003. 84(2): p. 205-14. 
18. Sikavitsas, V.I., G.N. Bancroft, and A.G. Mikos, Formation of three-dimensional cell/polymer constructs for bone tissue engineering in a spinner flask and a rotating wall vessel bioreactor. J Biomed Mater Res, 2002. 62(1): p. 136-48.

19. Song, K., et al., Three-dimensional fabrication of engineered bone with human bio-derived bone scaffolds in a rotating wall vessel bioreactor. J Biomed Mater Res A, 2008. 86(2): p. 323-32.

20. Sailon, A.M., et al., A novel flow-perfusion bioreactor supports $3 D$ dynamic cell culture. J Biomed Biotechnol, 2009. 2009: p. 873816.

21. Diederichs, S., et al., Dynamic cultivation of human mesenchymal stem cells in a rotating bed bioreactor system based on the Z RP platform. Biotechnol Prog, 2009. 25(6): p. 1762-71.

22. Frohlich, M., et al., Bone grafts engineered from human adipose-derived stem cells in perfusion bioreactor culture. Tissue Eng Part A, 2010. 16(1): p. 179-89.

23. Braccini, A., et al., Three-dimensional perfusion culture of human bone marrow cells and generation of osteoinductive grafts. Stem Cells, 2005. 23(8): p. 1066-72.

24. Scaglione, S., et al., Engineering of osteoinductive grafts by isolation and expansion of ovine bone marrow stromal cells directly on 3D ceramic scaffolds. Biotechnol Bioeng, 2006. 93(1): p. 181-7.

25. Gan, Y., et al., The clinical use of enriched bone marrow stem cells combined with porous beta-tricalcium phosphate in posterior spinal fusion. Biomaterials, 2008. 29(29): p. 3973-82.

26. Wongchuensoontorn, C., et al., Application of a new chair-side method for the harvest of mesenchymal stem cells in a patient with nonunion of a fracture of the atrophic mandible--a case report. J Craniomaxillofac Surg, 2009. 37(3): p. 15561.

27. D'Ippolito, G., et al., Age-related osteogenic potential of mesenchymal stromal stem cells from human vertebral bone marrow. J Bone Miner Res, 1999. 14(7): p. 1115-22.

28. Oyajobi, B.O., et al., Isolation and characterization of human clonogenic osteoblast progenitors immunoselected from fetal bone marrow stroma using STRO-1 monoclonal antibody. J Bone Miner Res, 1999. 14(3): p. 351-61.

29. Quirici, N., et al., Isolation of bone marrow mesenchymal stem cells by anti-nerve growth factor receptor antibodies. Exp Hematol, 2002. 30(7): p. 783-91.

30. Fennema, E.M., et al., The effect of bone marrow aspiration strategy on the yield and quality of human mesenchymal stem cells. Acta Orthop, 2009. 80(5): p. 61821.

31. Both, S.K., et al., A rapid and efficient method for expansion of human mesenchymal stem cells. Tissue Eng, 2007. 13(1): p. 3-9.

32. Yuan, H., et al., A comparison of the osteoinductive potential of two calcium phosphate ceramics implanted intramuscularly in goats. J Mater Sci Mater Med, 2002. 13(12): p. 1271-5.

33. Salgado, A.J., O.P. Coutinho, and R.L. Reis, Bone tissue engineering: state of the art and future trends. Macromol Biosci, 2004. 4(8): p. 743-65.

34. Roldan, J.C., et al., Bone formation and degradation of a highly porous biphasic calcium phosphate ceramic in presence of BMP-7, VEGF and mesenchymal stem cells in an ectopic mouse model. J Craniomaxillofac Surg, 2010. 
35. Hu, J.Z., et al., An ectopic study of tissue-engineered bone with Nell-1 gene modified rat bone marrow stromal cells in nude mice. Chin Med J (Engl), 2009. 122(8): p. 972-9.

36. Tasso, R., et al., The recruitment of two consecutive and different waves of host stem/progenitor cells during the development of tissue-engineered bone in a murine model. Biomaterials, 2010. 31(8): p. 2121-9.

37. Fernandes, H., et al., Endogenous Collagen Influences Differentiation of Human Multipotent Mesenchymal Stromal Cells. Tissue Eng Part A, 2010.

38. Meijer, G.J., et al., Cell-based bone tissue engineering. PLoS Med, 2007. 4(2): p. e9.

39. Kruyt, M.C., et al., Viable osteogenic cells are obligatory for tissue-engineered ectopic bone formation in goats. Tissue Eng, 2003. 9(2): p. 327-36.

40. Kadiyala, S., Culture-expanded, Bone marrow-derived Mesenchymal stem cells can regenerate a critical-sized Segmental Bone defect. Tissue engineering, 1997. 3(2).

41. Martin, I., T. Smith, and D. Wendt, Bioreactor-based roadmap for the translation of tissue engineering strategies into clinical products. Trends Biotechnol, 2009. 27(9): p. 495-502.

42. Braccini, A., et al., The osteogenicity of implanted engineered bone constructs is related to the density of clonogenic bone marrow stromal cells. J Tissue Eng Regen Med, 2007. 1(1): p. 60-5.

43. Banfi, A., et al., Proliferation kinetics and differentiation potential of ex vivo expanded human bone marrow stromal cells: Implications for their use in cell therapy. Exp Hematol, 2000. 28(6): p. 707-15.

44. Mendes, S.C., et al., Bone tissue-engineered implants using human bone marrow stromal cells: effect of culture conditions and donor age. Tissue Eng, 2002. 8(6): p. 911-20.

45. Alves, H., et al., A link between the accumulation of DNA damage and loss of multipotency of human mesenchymal stromal cells. J Cell Mol Med, 2009. 
Table 2 Donor information for optimization of volume of bone marrow to scaffold ratio

\begin{tabular}{|c|c|c|c|}
\hline Donor & Age & Sex & $\begin{array}{c}\text { Nucleated } \\
\text { cell/ml x } 10^{6}\end{array}$ \\
\hline 1 & 65 & Female & 8.3 \\
2 & 72 & female & 26.6 \\
3 & 66 & female & 15 \\
4 & 60 & Male & 28 \\
5 & 71 & Female & 25 \\
6 & 45 & Male & 10.4 \\
7 & 69 & Male & 15.4 \\
8 & 83 & Female & 17.8 \\
\hline
\end{tabular}




\section{Figure Legends}

\section{Fig 1. Cell proliferation and viability on porous ceramic scaffolds.}

Methylene blue staining of scaffolds seeded with unprocessed bone marrow after 1 (A), 2 (B) or 3 (C) weeks of culture. The blue dots represent cells on the scaffold. MTT staining at week 3 of a scaffold seeded with unprocessed bone marrow (D). As a control, cells from the same donor were expanded and seeded on the scaffold and after 1 week stained for methylene blue (E) and MTT (F). To verify that scaffold by itself does not stain with methylene blue or MTT, a representative scaffold without cells was stained for methylene blue $(\mathrm{G})$ and MTT $(\mathrm{H})$.

\section{Fig 2. Optimisation of volume of bone marrow to scaffold ratio}

Methylene blue staining of 1 scaffold from groups of 3 scaffolds seeded with $50 \mu \mathrm{l}$ (A), $100 \mu \mathrm{l}(\mathrm{B}), 200 \mu \mathrm{l}(\mathrm{C}), 400 \mu \mathrm{l}(\mathrm{D}), 800 \mu \mathrm{l}(\mathrm{E}), 1 \mathrm{ml}(\mathrm{F})$ of unprocessed bone marrow, after 3 weeks of culture. The blue dots represent cells on the scaffold while the arrow in 
Fig E denotes a cell clump. $200 \mu$ of bone marrow per 3 scaffolds gave optimum cell coverage on the scaffold surface while lower volumes did not form a cell sheath. Seeding higher volumes on the scaffolds resulted in the cell sheath detaching from the scaffold surface. Arrow in figure F denotes the detaching cell sheath.

\section{Fig 3. Quantification of cell growth.}

Estimation of DNA content to quantify the number of cells present on scaffolds cultured with either unprocessed bone marrow (DS) or 2D expanded cells (2D). In two of the three donors tested, the number of cells was similar in both groups. In donor 3, 2 times more cells were found on scaffolds seeded with 2D expanded cells as compared to those seeded directly with unprocessed bone marrow.

\section{Fig 4. Characterization of cells on ceramic scaffolds}

Immunostaining with CD45, CD31 and CD105 antibodies on ceramic scaffolds. Brown staining of membrane indicates cells positive for the antibody while blue dots represent the nucleus of the cells. Clusters of CD45 positive cells (A) and CD31 positive cells (B) on scaffolds seeded with unprocessed bone marrow. Both CD 45 and Cd 31 antibody staining was negative on scaffolds seeded with 2D expanded cells (C). On staining with CD105, a positively stained cell sheath is noted on scaffolds seeded with unprocessed bone marrow (D). Similar results were observed with scaffolds seeded with 2D expanded cells. On scanning electron microscopy of scaffolds seeded with 2D expanded cells, all cells had a similar morphology indicating presence of cells of the same lineage (E). 


\section{Fig 5. Bone formation on porous ceramic scaffolds}

(A). Representative histological section of scaffolds directly seeded with bone marrow and implanted in vivo for 6 weeks prior to staining with basic fuschin and methylene blue. This representative image shows newly formed bone (red with blue arrow), osteocytes embedded in matrix (black arrow) and the scaffold (white arrow). (B). Bar graph comparing the amount of bone formed by scaffolds seeded directly or with 2D expanded cells from the bone marrow of 4 donors. The data was analysed using Students paired T test. 
A

Methylene blue

Week 1

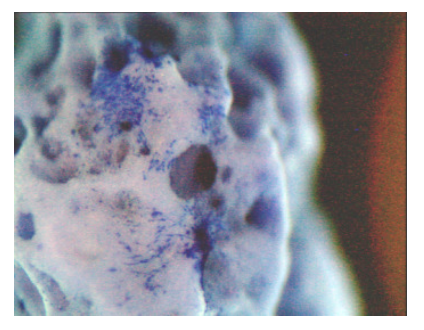

E

Methylene blue Week 3

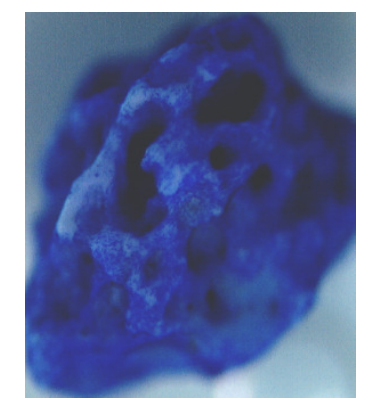

B

Methylene blue

Week 2

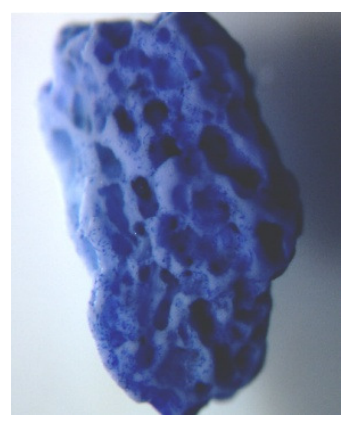

$\mathbf{F}$

MTT

Week 3

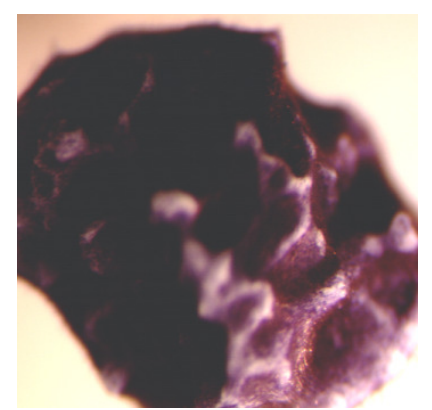

C

Methylene blue

Week 3

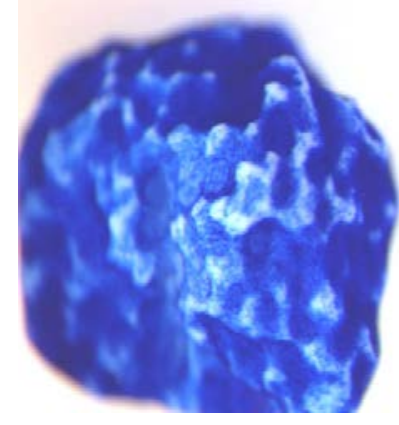

G

Methylene bllue Empty scaffold

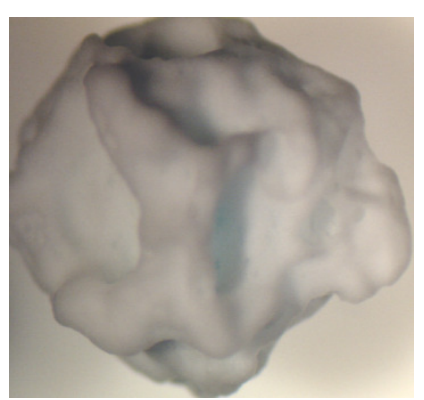

D

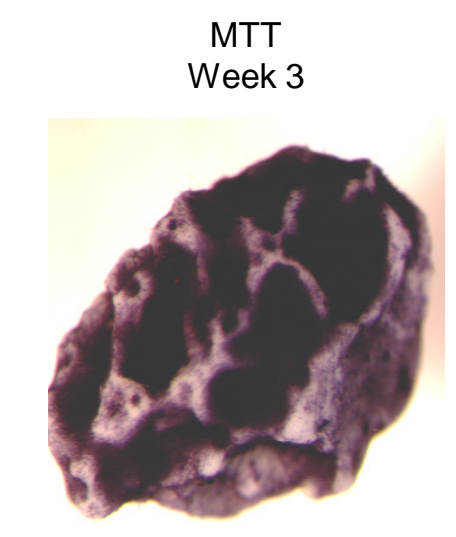

H

$$
\begin{gathered}
\text { MTT } \\
\text { Empty scaffold }
\end{gathered}
$$

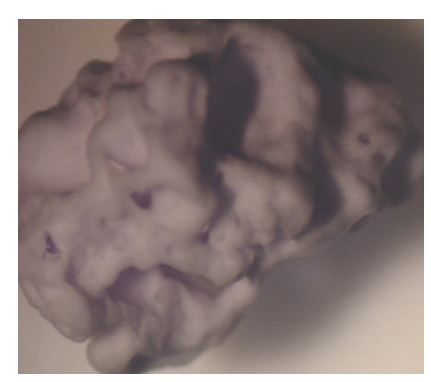

Figure 1 Chatterjea et al 

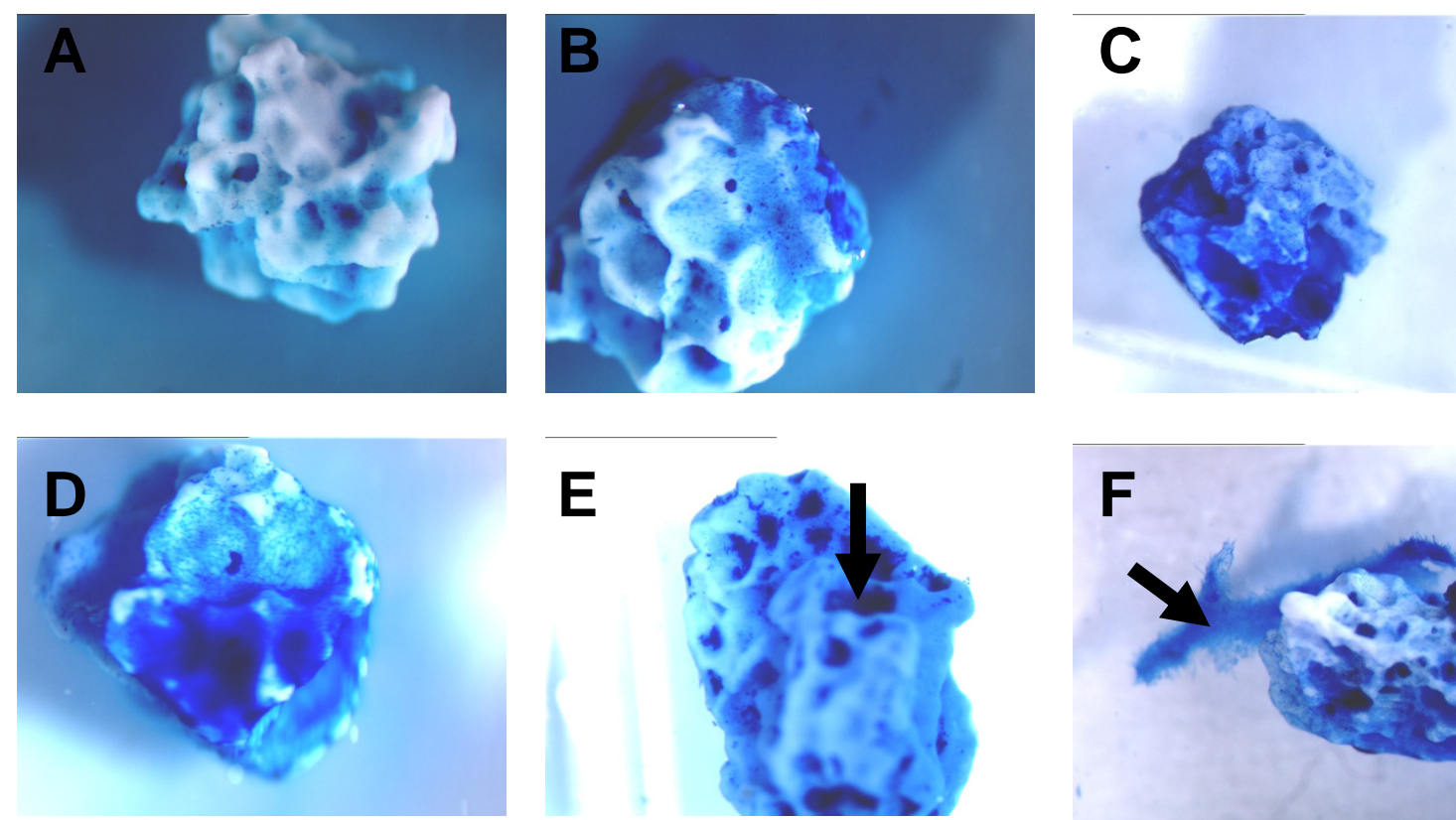

E
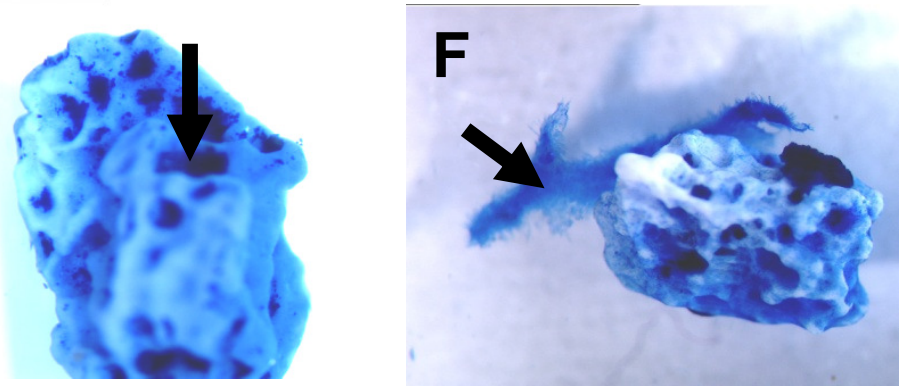

Figure 2 Chatterjea et al 


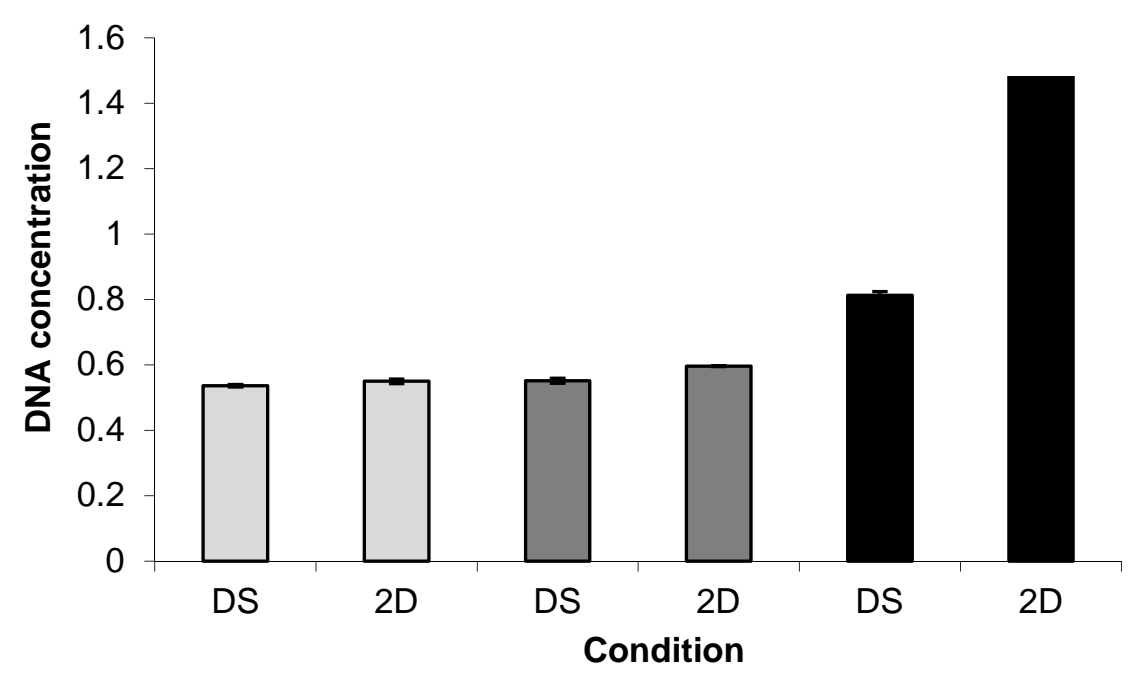

Donor 1

Donor 2

Donor 3

Figure 3 Chatterjea et al 


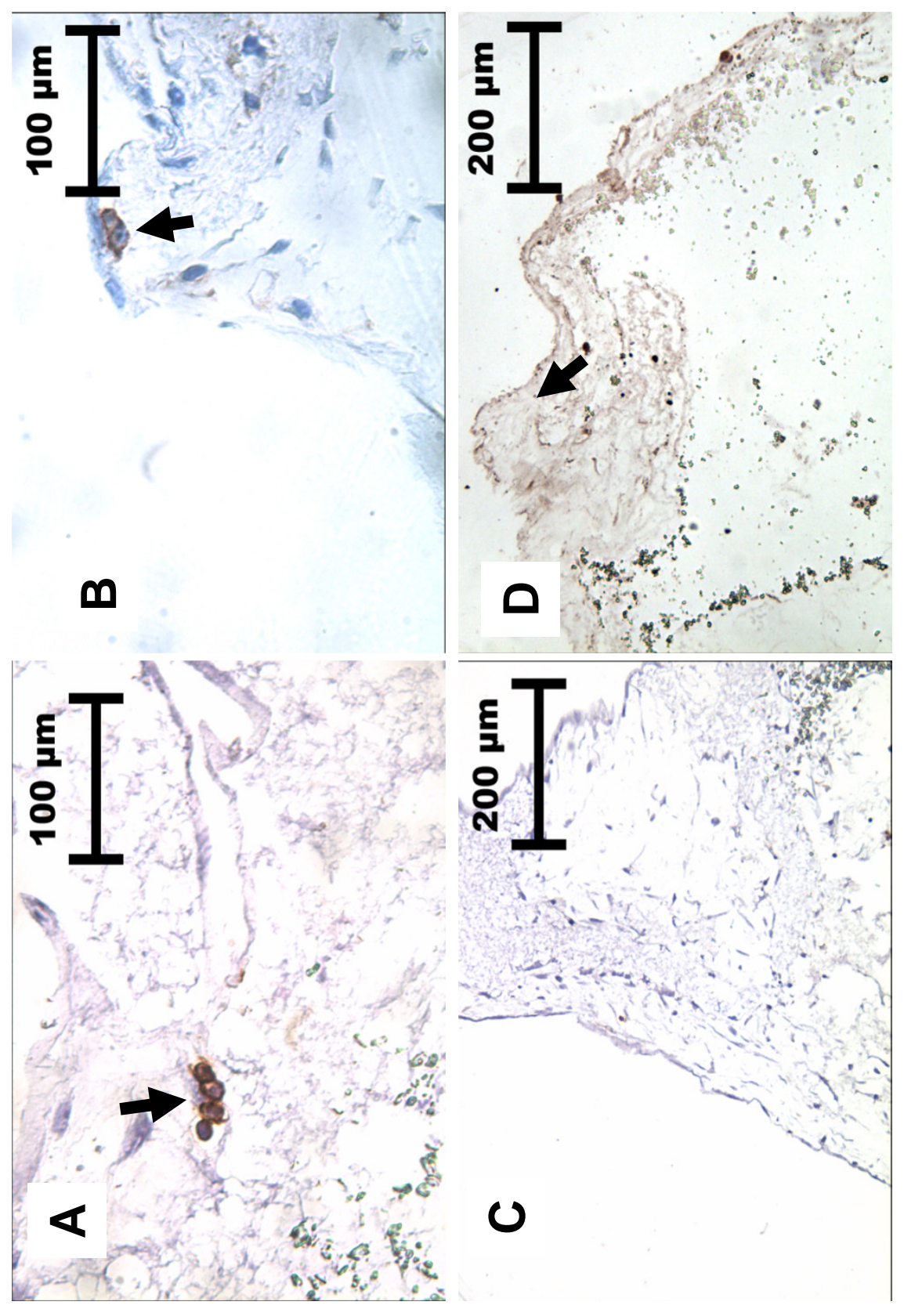

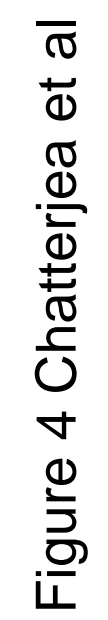

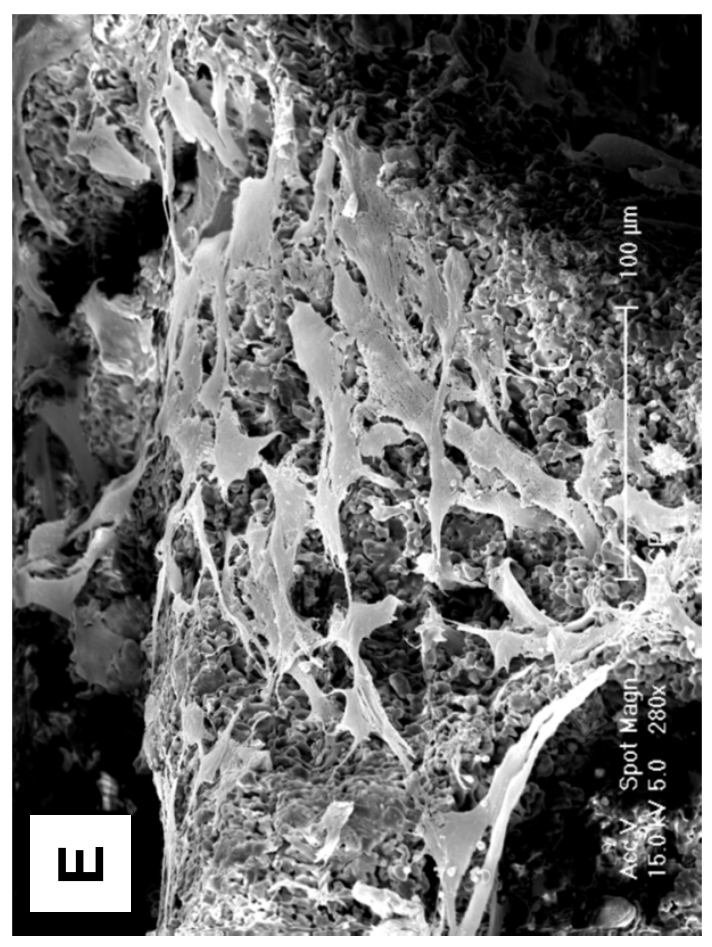


Figure 4

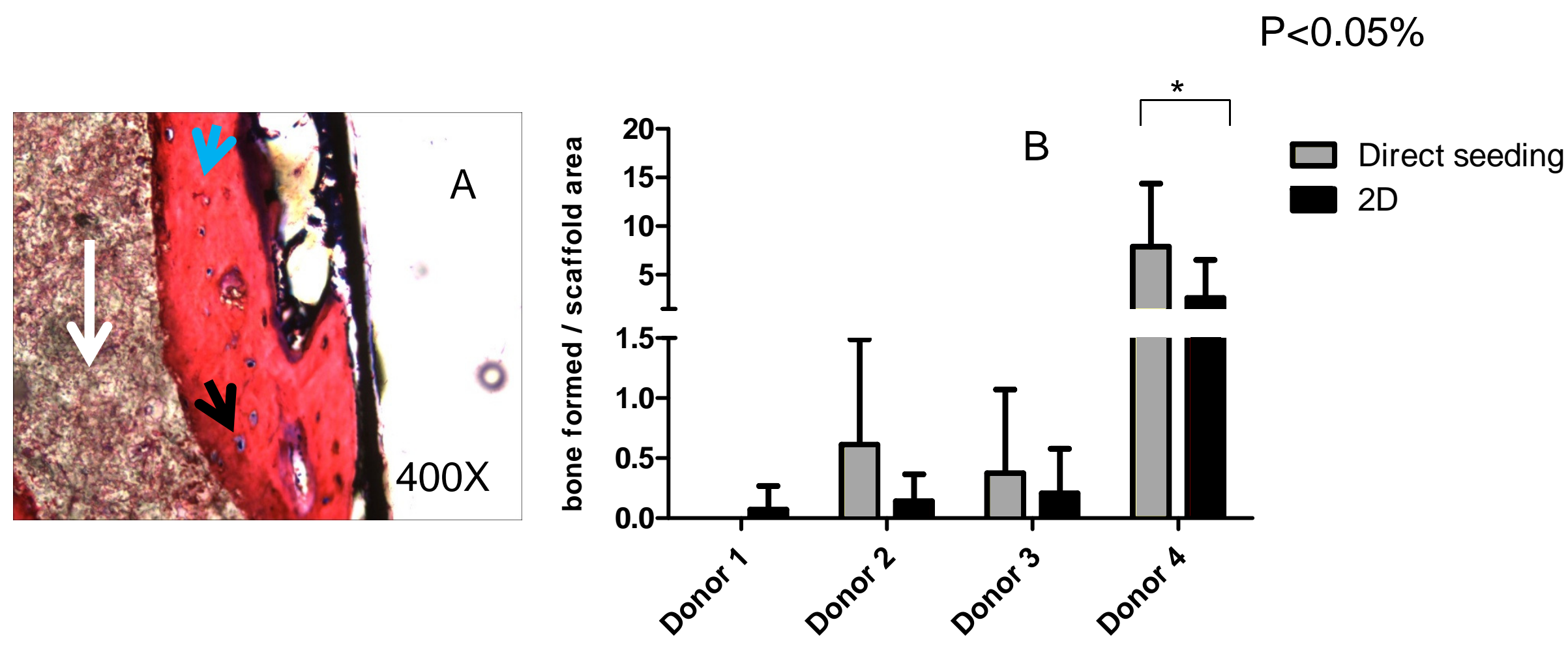

Figure 5 Chatterjea et al 